\title{
Galhas de insetos do Parque Estadual Paulo César Vinha (Guarapari, ES, Brasil)
}

\author{
Juliana de Menezes Bregonci ${ }^{1}$, Polyanna Vieira Polycarpo ${ }^{1}$ \& Valéria Cid Maia ${ }^{2,3}$ \\ ${ }^{1}$ Faculdades Integradas São Pedro, FAESA Campus II, \\ Av. Serafim Derenzi, s/n, São Pedro, Vitória, ES, Brasil \\ ${ }^{2}$ Departamento de Entomologia, Museu Nacional, \\ Universidade Federal do Rio de Janeiro - UFRJ, \\ Quinta da Boa Vista, São Cristovão, CEP 20940-049, Rio de Janeiro, RJ, Brasil \\ ${ }^{3}$ Autor para correspondência: Valéria Cid Maia, e-mail: maiavcid@acd.ufrj.br
}

BREGONCI, J.M., POLYCARPO, P.V. \& MAIA, V.C. Insect galls of the Parque Estadual Paulo César

Vinha (Guarapari, ES, Brazil). Biota Neotrop., 10(1): http://www.biotaneotropica.org.br/v10n1/en/ abstract?inventory+bn01410012010.

\begin{abstract}
Insect galls of the Parque Estadual Paulo César Vinha (Guarapari, ES, Brazil). Thirty-eigth morphotypes of insect galls were found on 21 plant species (19 genera and 17 families) in the Parque Estadual Paulo César Vinha (Guaparari, ES). The majority of the galls occurred on the leaves (84\%). Stem (13\%) and bud galls (3\%) were also found. One-chambered galls were the most frequent (52\%), as well as glabrous ones (89\%). Most part of them (84\%) was induced by Cecidomyiidae (Diptera). Hemipteran galls were also found (16\%). Parasitoid wasps (Hymenoptera) were obtained from 12 gall morphotypes, being represented by Eurytomidae, Eulophidae, Platygasteridae, Pteromalidae, and Torymidae. Inquilines were found in three morphotypes, being represented by Coleoptera, Lepidoptera, and Thysanoptera. Predaceous ants (Formicidae, Hymenoptera) were obtained from a single morphotype. Acarii and Psocoptera were found as sucessores in three morphotypes. Nyctaginaceae, Myrtaceae, Sapotaceae, Lauraceae, and Smilaceae were the richest plant families in number of galls. Five new records of host plants are present: Emmotum nitens (Icacinaceae), Andira nitida (Fabaceae), Chaetocarpus myrsinites (Euphorbiaceae), Salzmania nitida DC. (Rubiaceae), and Epidendrum denticulatum (Orchidaceae). Three new records of insect galls on three plant species - Clusia hilariana (Clusiaceae), Guapira pernambuscensis (Nyctaginaceae), and Ouratea cuspidata (Ochnaceae) are present. All records are new for the Espírito Santo State.
\end{abstract}

Keywords: galling species, host plant, Cecidomyiidae, Diptera, restinga.

BRegonCI, J.M., POLYCARPO, P.V. \& MAIA, V.C. Galhas de insetos do Parque Estadual Paulo César Vinha (Guarapari, ES, Brasil). Biota Neotrop., 10(1): http://www.biotaneotropica.org.br/v10n1/pt/ abstract?inventory+bn01410012010.

Resumo: Galhas de insetos do Parque Estadual Paulo César Vinha (Guarapari, ES, Brasil). Trinta e oito morfotipos de galhas de insetos foram encontradas em 21 espécies de planta (19 gêneros e 17 famílias) no Parque Estadual Paulo César Vinha (Guaparari, ES). A maioria das galhas ocorreu em folhas (84\%). Galhas caulinares (13\%) e das gemas (3\%) também foram encontradas. Galhas uniloculares foram mais frequientes (52\%), assim como as glabras (89\%). A maioria delas (84\%) foi induzida por Cecidomyiidae (Diptera). Galhas de Hemiptera também foram encontradas (16\%). Vespas parasitóides (Hymenoptera) foram obtidas de 22 morfotipos de galha, estando representadas por Eurytomidae, Eulophidae, Platygasteridae, Pteromalidae e Torymidae. Inquilinos foram encontrados em três morfotipos, estando representados por Coleoptera, Lepidoptera e Thysanoptera. Formigas predadoras (Formicidae, Hymenoptera) foram obtidas de um único morfotipo. Acarii e Psocoptera foram encontrados como sucessores em três morfotipos. Nyctaginaceae, Myrtaceae, Sapotaceae, Lauraceae e Smilaceae foram as famílias de planta com maior riqueza de galhas. Cinco novos registros de plantas hospedeiras são apresentados: Emmotum nitens (Icacinaceae), Andira nitida (Fabaceae), Chaetocarpus myrsinites (Euphorbiaceae), Salzmania nitida DC. (Rubiaceae) e Epidendrum denticulatum (Orchidaceae). Três novos registros de galhas de insetos em três espécies de planta - Clusia hilariana (Clusiaceae), Guapira pernambuscensis (Nyctaginaceae) e Ouratea cuspidata (Ochnaceae) são assinalados. Todos os registros apresentados são novos para o estado do Espírito Santo.

Palavras-chave: galhadores, planta hospedeira, Cecidomyiidae, Diptera, restinga. 


\section{Introdução}

As restingas se estendem por quase toda costa brasileira, numa extensão total de aproximadamente cinco mil quilômetros, sendo as principais formações encontradas entre a Bahia e São Paulo (Neiman 1989). Compreendem uma grande variedade de comunidades vegetais, devido à diversidade topográfica das condições ambientais (Araújo \& Henriques 1984), e apresentam uma grande riqueza de galhas de insetos, provavelmente devido ao estresse higrotérmico causado pelos contrastes diários de temperatura, umidade, rajadas de ventos, forte radiação e solo arenoso (Monteiro et al. 1994, Maia 2001, Monteiro et al. 2004).

No Sudeste brasileiro, áreas de restinga do estado do Rio de Janeiro (Maricá, Carapebus, Arraial do Cabo e Grumari) e de São Paulo (Bertioga) foram investigadas com relação à riqueza de galhas de insetos. Esses estudos apontaram as Myrtaceae, Burseraceae, Nyctaginaceae, Erythroxylaceae, Lauraceae e Melastomataceae e Asteraceae como as famílias de planta com maior riqueza de galhas e corroboraram os Cecidomyiidae como o principal táxon galhador (Monteiro et al. 1994, Maia 2001a, Monteiro et al. 2004, Oliveira \& Maia 2005, Maia et al. 2008).

Uma rica entomofauna encontra-se associada às galhas, e inclui predadores, parasitóides, inquilinos e sucessores, de forma que as galhas representam um verdadeiro microhabitat, onde diversas relações tri-tróficas são estabelecidas (Maia 2001a).

Espécies galhadoras e a entomofauna associada podem ser utilizadas como indicadoras da qualidade ambiental, sendo ferramentas úteis na avaliação do impacto humano nesses ambientes tão ameaçados. Como bioindicadores respondem às perturbações ambientais mostrando perda de diversidade e alterações nas abundâncias populacionais tanto das espécies galhadoras como dos parasitóides (Oliveira 2009).
Para o estado do Espírito Santo, não há informações sobre a diversidade de galhas de insetos, sendo esta, portanto, a primeira contribuição para o seu conhecimento.

\section{Material e Métodos}

A coleta de dados foi realizada no Parque Estadual Paulo César Vinha (PEPCV), em Setiba, Guarapari, estado do Espírito Santo. Esse Parque possui 1.500 ha e situa-se entre as coordenadas $20^{\circ} 33^{\prime}$ $20^{\circ} 38^{\prime} \mathrm{S}$ e $40^{\circ} 23^{\prime}-40^{\circ} 26^{\prime} \mathrm{O}$. O clima da região é do tipo Aw, segundo classificação de Koeppen, apresentando temperatura média anual de $23,3{ }^{\circ} \mathrm{C}$, precipitação média anual de $1.307 \mathrm{~mm}$ e umidade relativa média anual de 80\% (Fabris 1995).

As coletas foram realizadas uma vez por mês, no período de julho de 2006 a outubro de 2007, de forma a possibilitar acompanhamento da variação sazonal. Dentre as diversas comunidades vegetais do PEPCV, foi escolhida uma área de formação aberta não inundável, em função da facilidade de deslocamento e acessibilidade da vegetação. As formações abertas não inundáveis ocorrem em áreas não inundadas, cujo lençol freático situa-se, em geral, a dois metros abaixo da superfície do solo, podendo chegar a sete metros em algumas restingas, dependendo da topografia e da época do ano. É característica marcante a presença de areia branca exposta e a vegetação distribuída em moitas de diferentes tamanhos e formas, com até oito metros de altura, intercaladas por espaços onde a cobertura vegetal é esparsa (Araújo \& Henriques 1984). Para amostragem, foi utilizado o método de intercepto de linha (Brower \& Zar 1984), com 12 linhas de $50 \mathrm{~m}$, espaçadas por intervalos de $10 \mathrm{~m}$, perfazendo um total de $600 \mathrm{~m}$. A vegetação de cada intercepto foi vistoriada à procura de galhas de insetos. Visando a identificação das plantas hospedeiras, foram feitas exsicatas de ramos com flores e/ou frutos de acordo com as

Tabela 1. Distribuição dos morfotipos de galhas de inseto por famílias e espécie de planta hospedeira na restinga do Parque Estadual Paulo César Vinha Setiba, Guarapari, ES, no período de julho, 2006 a outubro, 2007.

Table 1. Distribution of the morphotypes of insetc galls per family and species of host plant in the restinga of the Parque Estadual Paulo César Vinha, Setiba, Guarapari, ES, from July, 2006 to October, 2007.

\begin{tabular}{|c|c|c|}
\hline Famílias & Espécies & $\mathbf{N}^{\circ}$ de morfotipos \\
\hline APOCYNACEAE & Não identificada & 2 \\
\hline AQUIFOLIACEAE & Ilex sp. & 1 \\
\hline BORAGINACEAE & Cordia verbenacea $D C$. & 1 \\
\hline BURSERACEAE & Protium icicariba (DC.) Marchand & 2 \\
\hline CHRYSOBALANACEAE & Couepia ovalifolia (Schott) Benth. & 2 \\
\hline CLUSIACEAE & Clusia hilariana Schltdl. & 2 \\
\hline EUPHORBIACEAE & Chaetocarpus myrsinites Baill. & 2 \\
\hline FABACEAE & Andira nitida Mart. ex Benth & 3 \\
\hline LAURACEAE & Ocotea notata (Nees) Mez & 1 \\
\hline MALPIGHIACEAE & Byrsonima sericea DC. & 2 \\
\hline MYRTACEAE & Neomitranthes obscura Sobral \& Zambom & 1 \\
\hline \multirow{5}{*}{ NYCTAGINACEAE } & Myrciaria floribunda (H. West ex Willd.) Legrand & 3 \\
\hline & Não identificada & 1 \\
\hline & Guapira pernambuscensis (Casar.) Lundell & 3 \\
\hline & Guapira opposita (Vell.) Reitz & 1 \\
\hline & Guapira sp. & 1 \\
\hline OCHNACEAE & Ouratea cuspidata (A. St.-Hil.) Engl. & 2 \\
\hline ORCHIDACEAE & Epidendrum denticulatum Barb. Rodr. & 1 \\
\hline RUBIACEAE & Salzmania nitida DC. & 1 \\
\hline SAPOTACEAE & Manilkara subsericea (Mart.) Dubard & 4 \\
\hline SMILACEAE & Smilax rufescens Griseb. & 2 \\
\hline
\end{tabular}


técnicas usuais (Fidalgo \& Bononi 1989). A identificação baseou-se na literatura especializada, comparação com material depositado em herbários e envio para especialistas (quando necessário).

As galhas foram caracterizadas morfologicamente quanto à coloração, forma, órgão de ocorrência na planta hospedeira, número de câmaras internas e presença ou ausência de tricomas. No caso de galhas foliares, foi assinalada a sua ocorrência na face adaxial ou abaxial, no limbo e/ou nas nervuras (principais e/ou secundárias). Com relação à coloração, foi verificada se era similar ou distinta da coloração do órgão vegetal de ocorrência.

Para a obtenção da entomofauna associada, amostras de cada morfotipo de galha foram coletadas e transportadas em sacos e potes plásticos vedados, umedecidos e etiquetados (Almeida et al. 2003). No laboratório, parte da amostra foi dissecada com auxílio de estereomicroscópio para a retirada das larvas e pupas encontradas no interior das galhas. Para a obtenção dos adultos, amostras de cada morfotipo de galha foram acondicionadas separadamente em potes plásticos vedados, etiquetados e vistoriados diariamente. Os insetos encontrados nas galhas foram classificados quanto a seus hábitos em galhadores, inquilinos, sucessores (inquilinos de galhas já abandonadas pelo galhador) e predadores, com base em observações do conteúdo das câmaras internas e em consultas bibliográficas.

Todos os insetos obtidos foram inicialmente conservados em álcool etílico 70\%. Para a identificação dos Cecidomyiidae (Diptera), as técnicas de montagem foram baseadas em Gagné (1994). Para a identificação dos gêneros, as chaves de Gagné (1994) foram utilizadas. As espécies foram identificadas com base nas descrições originais e por comparação com o material depositado na coleção de Diptera do Museu Nacional (MNRJ/UFRJ).

Todo o material estudado encontra-se depositado na Coleção entomológica da Universidade Federal do Espírito Santo (UFES), e as exsicatas foram incorporadas ao acervo do Herbário VIES (UFES).

\section{Resultados}

Foram encontrados 38 morfotipos de galhas em 21 espécies de plantas distribuídas em 19 gêneros e 17 famílias. A média de morfotipos de galhas por espécie de planta hospedeira foi de 1,8.

As famílias que apresentaram maior riqueza de galhas foram Nyctaginaceae, Myrtaceae, Sapotaceae, Lauraceae e Smilaceae, com sete, cinco, quatro, quatro e três morfotipos, respectivamente (Tabela 1). A ocorrência de galhas em Andira nitida Mart. ex Benth (Fabaceae), Chaetocarpus myrsinites Baill. (Euphorbiaceae),
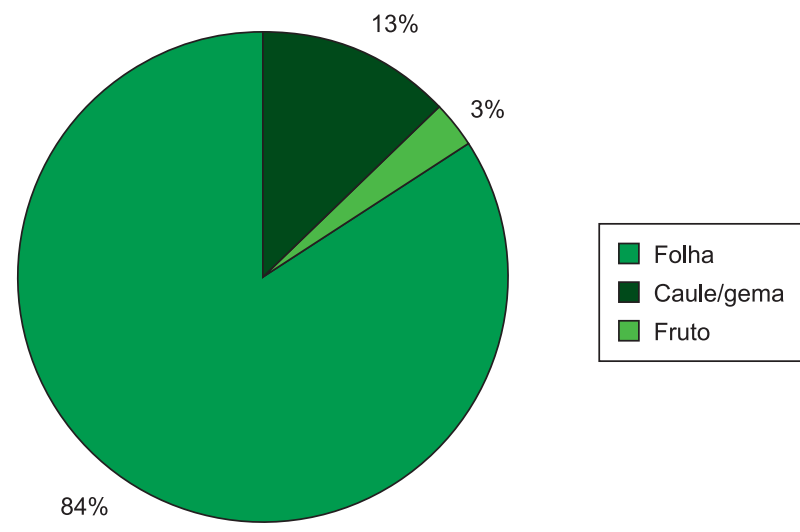

Figura 1. Distribuição do número de morfotipos de galhas de insetos nos órgãos vegetais no Parque Estadual Paulo César Vinha, Guarapari, ES.

Figure 1. Distribution of the number of morphotypes of insect galls per plant organ in the Parque Estadual Paulo César Vinha, Guarapari, ES.
Emmotum nitens (Icacinaceae), Epidendrum denticulatum Barb. Rodr.(Orchidaceae) e Salzmania nitida DC. (Rubiaceae) é assinalada pela primeira vez. Não foram observadas galhas em Cactaceae, Bromeliaceae, Araceae e Arecaceae, embora essas plantas tenham sido investigadas.

Foram encontradas galhas em folhas $(84 \%)$, caules ou gemas (13\%) e frutos (3\%), sendo que cada morfotipo ocorreu em um único órgão da planta hospedeira (Figura 1). Nenhuma galha foi observada em flores, raízes aéreas e gavinhas, embora tais órgãos tenham sido vistoriados.

Com relação à forma, observamos enrolamentos da borda foliar, galhas globosas, redondas, cônicas, circulares, triangulares, elípticas e ovóides. As galhas globosas, os enrolamentos da borda da folha e as galhas redondas predominaram, com 28, 23 e $16 \%$ do total, respectivamente (Figura 2).

A maioria das galhas exibiu a mesma coloração do órgão de ocorrência. A cor verde predominou nas galhas foliares, porém foi também observada em galhas caulinares em duas espécies de plantas: Manilkara subsericea e Myrciaria floribunda (H. West ex Willd.) Legrand. A coloração marrom predominou em galhas caulinares, sendo observada também em galhas foliares em seis espécies de plantas: Chaetocarpus myrsinites, Epidendrum denticulatum, Guapira sp., Ilex sp., Ouratea cuspidata e Protium icicariba (DC.). A cor rosa ocorreu em galhas de diversos órgãos (folha, caule e fruto), sendo sempre distinta da coloração do órgão de ocorrência da galha (Figura 3). Algumas galhas, no decorrer do período de seu desenvolvimento, sofreram alterações de coloração, como por exemplo, a galha globosa foliar em Myrciaria floribunda, inicialmente verde, e com o passar do tempo, adquiriu coloração amarelo-avermelhada.

Com relação à ornamentação das galhas, observou-se que apenas $11 \%$ do total dos morfotipos possuíam pêlos ou tricomas em sua estrutura, e os outros $89 \%$ eram glabros.

Foi verificada maior porcentagem de galhas uniloculares (52\%). Em alguns morfotipos, o número de câmaras internas variou de um e dois $(11 \%)$, um a três $(8 \%)$ e três a quatro (3\%). Outros morfotipos possuíam mais de dez câmaras (8\%). Contudo, em 18\% das galhas não foi possível quantificar o número de câmaras internas, uma vez que encontravam-se já abandonadas pelo indutor e em deterioração.

As galhas foram induzidas por Diptera e Hemiptera. Todos os Diptera galhadores pertencem à família Cecidomyiidae. Não foram encontradas galhas induzidas por Thysanoptera, Lepidoptera, Coleoptera e Hymenoptera. Devido à escassez de material, dezenove indutores não puderam ser determinados (Tabela 2).

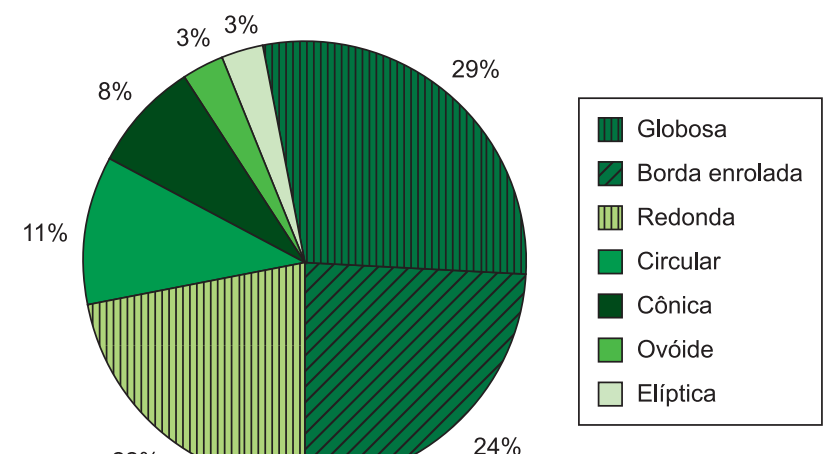

Figura 2. Distribuição dos morfotipos de galhas entomógenas encontradas na restinga do Parque Estadual Paulo César Vinha, Guarapari, ES.

Figure 2. Distribution of the morphotypes of insect galls found in the restinga of the Parque Estadual Paulo César Vinha, Guarapari, ES. 


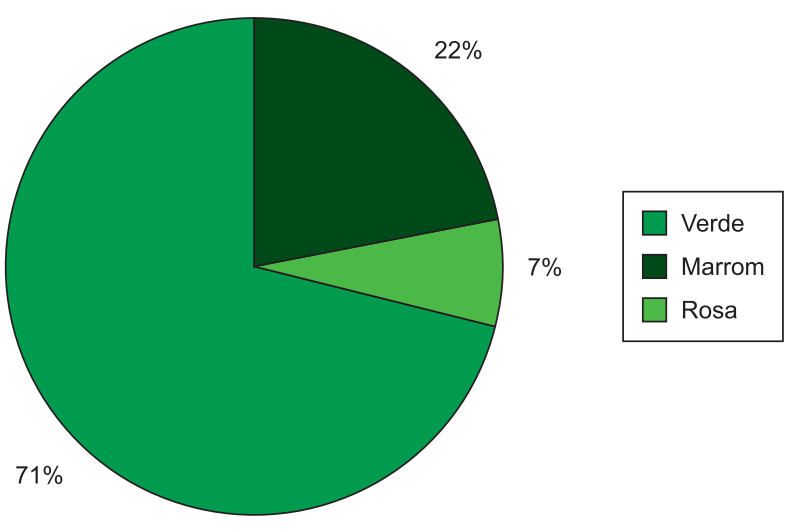

Figura 3. Frequiência das cores das galhas de insetos na restinga do Parque Estadual Paulo César Vinha, Guarapari, ES.

Figure 3. Frequency of the colors of the insect galls in the restinga of the Parque Estadual Paulo César Vinha, Guarapari, ES.
Dentre os Cecidomyiidae galhadores, seis foram identificados em nível de espécie (Lopesia simplex Maia, 2002; Dasineura couepiae Maia, 2001; Clusiamyia granulosa Maia, 1996; Dasineura myrciariae Maia 1993; Manilkaramyia notabilis Maia, 2001 e Cordiamyia globosa Maia, 1996), dois em nível de gênero: Bruggmannia sp. e Contarinia sp. e os demais em família.

Dentre os hemípteros galhadores, apenas um foi identificado em nível de família, sendo os demais identificados em ordem.

A fauna de artrópodes associada às galhas incluiu colêmbolos, ácaros, Hymenoptera, Coleoptera, Lepidoptera, Thysanoptera, Diptera, Hemiptera e Psocoptera. A ocorrência de microhimenópteros parasitóides foi verificada em doze morfotipos de galhas (cerca de $31 \%$ do total), representados por cinco famílias de Hymenoptera: Eurytomidae, Eulophidae, Platygasteridae, Pteromalidae, Torymidae (Tabela 2).

A presença de inquilinos foi observada em quatro morfotipos de galhas induzidos por Cecidomyiidae, cerca de $10 \%$ do total.

Tabela 2. Caracterização das galhas entomógenas (órgão da planta, forma, coloração, presença de tricomas, número de câmaras internas, indutor e fauna associada) encontradas na restinga do Parque Estadual Paulo César Vinha, Guarapari, ES, no período de julho, 2006 a outubro, 2007.

Tabela 2. Characterization of the insect galls (plant organ, shape, color, presence of trichomas, number of internal chambers, galler and associated fauna) found in the restinga of the Parque Estadual Paulo César Vinha, Guarapari, ES, from July, 2006 to October, 2007.

\begin{tabular}{|c|c|c|c|c|c|c|c|}
\hline $\begin{array}{c}\text { Planta } \\
\text { hospedeira }\end{array}$ & Órgão & Forma & Cor & Tricomas & $\begin{array}{l}\mathrm{N}^{\circ} \text { de } \\
\text { câm. }\end{array}$ & Indutor & $\begin{array}{c}\text { Fauna } \\
\text { associada }\end{array}$ \\
\hline \multirow[t]{2}{*}{ APOCYNACEAE } & Folha & Cônica & Verde & Ausente & 1 & Não det. & - \\
\hline & & Borda enrolada & Verde & Ausente & 1 & & \\
\hline \multirow[t]{3}{*}{ Andira nitida } & Folha & $\begin{array}{l}\text { Globosa } \\
\text { (Figura 5) }\end{array}$ & Verde & Ausente & $3-4$ & Não det. & $\begin{array}{l}\text { Entedoninae sp. } 2 \\
\text { Pteromalidae } \\
\text { Platygastridae sp. } 1\end{array}$ \\
\hline & & $\begin{array}{l}\text { Circular } \\
\text { (Figura 6) }\end{array}$ & Verde & Ausente & $1-3$ & Cecidomyiidae & $\begin{array}{l}\text { Coleoptera } \\
\text { Entedoninae sp. } 3 \\
\text { Entedoninae sp. } 4 \\
\text { Eurytomidae }\end{array}$ \\
\hline & & $\begin{array}{l}\text { Borda enrolada } \\
\text { (Figura 7) }\end{array}$ & Verde & Ausente & 1 & Cecidomyiidae & Hymenoptera \\
\hline \multirow[t]{2}{*}{ Byrsonima sericea } & Folha & $\begin{array}{l}\text { Circular } \\
\text { (Figura 8) }\end{array}$ & Marrom & Ausente & - & Não det. & - \\
\hline & Caule & $\begin{array}{l}\text { Globosa } \\
\text { (Figura 9) }\end{array}$ & Marrom & Ausente & - & Não det. & $\begin{array}{l}\text { Prodecatoma sp. } 1 \\
\text { (Eurytomidae) }\end{array}$ \\
\hline \multirow[t]{2}{*}{$\begin{array}{l}\text { Chaetocarpus } \\
\text { myrsinites }\end{array}$} & Folha & $\begin{array}{l}\text { Redonda } \\
\text { (Figura 10) }\end{array}$ & $\begin{array}{l}\text { Verde/ } \\
\text { Marrom }\end{array}$ & Ausente & $1-2$ & Não det. & $\begin{array}{l}\text { Cecidomyiidae } \\
\text { Lepidoptera } \\
\text { Psocoptera }\end{array}$ \\
\hline & & Borda enrolada & Marrom & Ausente & - & Hemiptera & - \\
\hline Clusia hilariana & Folha & $\begin{array}{l}\text { Circular } \\
\text { (Figura 11) }\end{array}$ & Verde & Ausente & $1-2$ & $\begin{array}{l}\text { Clusiamyia granulosa } \\
\text { Maia, } 1996 \\
\text { (Cecidomyiidae) }\end{array}$ & Torymidae sp.1 \\
\hline Clusia hilariana & Folha & $\begin{array}{l}\text { Borda enrolada } \\
\text { (Figura 12) }\end{array}$ & Verde & Ausente & - & Não det. & - \\
\hline Cordia verbenacea & Folha & $\begin{array}{l}\text { Globosa } \\
\text { (Figura 13) }\end{array}$ & Verde & Presente & 1 & $\begin{array}{l}\text { Cordiamyia globosa } \\
\text { Maia }\end{array}$ & - \\
\hline \multirow[t]{2}{*}{ Couepia ovalifolia } & Folha & $\begin{array}{l}\text { Redonda } \\
\text { (Figura 14) }\end{array}$ & Verde & Ausente & 1 & $\begin{array}{l}\text { Dasineura couepiae } \\
\text { Maia, } 2001 \\
\text { (Cecidomyiidae) }\end{array}$ & - \\
\hline & & Ovóide & Verde & Ausente & $1-2$ & Não det. & - \\
\hline $\begin{array}{l}\text { Epidendrum } \\
\text { denticulatum }\end{array}$ & Folha & $\begin{array}{l}\text { Circular } \\
\text { (Figura 15) }\end{array}$ & Marrom & Ausente & 1 & Não det. & - \\
\hline Guapira opposita & Folha & Globosa & Verde & Ausente & 1 & Não det. & - \\
\hline $\begin{array}{l}\text { Guapira } \\
\text { pernambuscensis }\end{array}$ & Folha & $\begin{array}{l}\text { Globosa } \\
\text { (Figura 16) }\end{array}$ & Rosa & Presente & 1 & $\begin{array}{l}\text { Bruggmannia sp. } \\
\text { (Cecidomyiidae) }\end{array}$ & Platygasteridae sp. 1 \\
\hline
\end{tabular}


Tabela 2. Continuação...

\begin{tabular}{|c|c|c|c|c|c|c|c|}
\hline $\begin{array}{c}\text { Planta } \\
\text { hospedeira }\end{array}$ & Órgão & Forma & Cor & Tricomas & $\begin{array}{l}\mathrm{N}^{\circ} \mathrm{de} \\
\text { câm. }\end{array}$ & Indutor & $\begin{array}{c}\text { Fauna } \\
\text { associada }\end{array}$ \\
\hline & & $\begin{array}{l}\text { Redonda } \\
\text { (Figura17) }\end{array}$ & Verde & Ausente & $1-3$ & Cecidomyiidae & $\begin{array}{l}\text { Lepidoptera } \\
\text { Ácaros } \\
\text { Rileya } \text { sp.1 } \\
\text { (Eurytomidae) } \\
\text { Entedoninae } \\
\text { (Eulophidae) } \\
\text { Tetrastichinae sp.1 } \\
\text { (Eulophidae) } \\
\text { Platygasteridae sp.1 } \\
\text { Formicidae sp.1 }\end{array}$ \\
\hline & Caule & $\begin{array}{l}\text { Globosa } \\
\text { (Figura 18) }\end{array}$ & Rosa & Presente & $1-2$ & Cecidomyiidae & Ácaros \\
\hline Guapira sp. & Folha & $\begin{array}{l}\text { Cônica } \\
\text { (Figura 19) }\end{array}$ & $\begin{array}{l}\text { Verde/ } \\
\text { Marrom }\end{array}$ & Ausente & 1 & Não det. & Entedoninae sp.4 \\
\hline Ilex sp. & Folha & $\begin{array}{l}\text { Redonda } \\
\text { (Figura 20) }\end{array}$ & $\begin{array}{l}\text { Verde } \\
\text { Marrom }\end{array}$ & Ausente & 1 & Cecidomyiidae & - \\
\hline \multirow[t]{4}{*}{$\begin{array}{l}\text { Manilkara } \\
\text { subsericea }\end{array}$} & Folha & $\begin{array}{l}\text { Elíptica } \\
\text { (Figura 21) }\end{array}$ & Verde & Ausente & 1 & $\begin{array}{l}\text { Manilkaramyia } \\
\text { notabilis } \\
\text { Maia, } 2001 \\
\text { (Cecidomyiidae) }\end{array}$ & - \\
\hline & & $\begin{array}{l}\text { Redonda } \\
\text { (Figura 22) }\end{array}$ & Verde & Ausente & 1 & Não det. & Hymenoptera \\
\hline & & Borda enrolada & Verde & Ausente & 1 & Não det. & - \\
\hline & Caule & $\begin{array}{l}\text { Globosa } \\
\text { (Figura 23) }\end{array}$ & Verde & Ausente & 10 & Cecidomyiidae & $\begin{array}{l}\text { Coleoptera } \\
\text { Hymenoptera }\end{array}$ \\
\hline \multirow[t]{3}{*}{$\begin{array}{l}\text { Myrciaria } \\
\text { floribunda }\end{array}$} & Folha & $\begin{array}{l}\text { Globosa } \\
\text { (Figura 24) }\end{array}$ & Verde & Presente & $1-3$ & Cecidomyiidae & $\begin{array}{l}\text { Thysanoptera } \\
\text { Prodecatoma } \mathrm{sp} .1 \\
\text { Formicidae sp. } 3\end{array}$ \\
\hline & & $\begin{array}{l}\text { Borda enrolada } \\
\text { (Figura 25) }\end{array}$ & Verde & Ausente & & $\begin{array}{l}\text { Dasineura } \\
\text { myrciariae } \\
\text { Maia, } 1993 \\
\text { (Cecidomyiidae) }\end{array}$ & - \\
\hline & Caule & $\begin{array}{l}\text { Globosa } \\
\text { (Figura 26) }\end{array}$ & Verde & Ausente & 1 & Não det. & - \\
\hline MYRTACEAE & Caule & $\begin{array}{l}\text { Globosa } \\
\text { (Figura 27) }\end{array}$ & Marrom & Ausente & 17 & Não det. & - \\
\hline $\begin{array}{l}\text { Neomitranthes } \\
\text { obscura }\end{array}$ & Folha & Borda enrolada & Verde & Ausente & - & Não det. & - \\
\hline Ocotea notata & Folha & $\begin{array}{l}\text { Redonda } \\
\text { (Figura 28) }\end{array}$ & Verde & Ausente & 1 & Hemiptera & $\begin{array}{l}\text { Hemiptera } \\
\text { Cecidomyiidae }\end{array}$ \\
\hline \multirow[t]{2}{*}{ Ouratea cuspidate } & Folha & $\begin{array}{l}\text { Triangular } \\
\text { (Figura 29) }\end{array}$ & Marrom & Ausente & 1 & $\begin{array}{l}\text { Contarinia sp. } \\
\text { (Cecidomyiidae) }\end{array}$ & Coleoptera \\
\hline & & Borda enrolada & Verde & Ausente & 1 & Não det. & - \\
\hline \multirow[t]{2}{*}{ Protium icicariba } & Folha & $\begin{array}{l}\text { Redonda } \\
\text { (Figura 30) }\end{array}$ & $\begin{array}{l}\text { Verde/ } \\
\text { marrom }\end{array}$ & Ausente & - & $\begin{array}{l}\text { Psyllidae } \\
\text { (Hemiptera) }\end{array}$ & - \\
\hline & & Borda Enrolada & Verde & Ausente & 1 & $\begin{array}{l}\text { Lopesia simplex Maia, } \\
2002 \\
\text { (Cecidomyiidae) }\end{array}$ & - \\
\hline Salzmania nitida & Fruto & $\begin{array}{l}\text { Globosa } \\
\text { (Figura 31) }\end{array}$ & Rosa & Ausente & 10 & Não det. & - \\
\hline \multirow[t]{2}{*}{ Smilax rufescens } & Folha & $\begin{array}{l}\text { Cônica } \\
\text { (Figura 32) }\end{array}$ & Verde & Ausente & - & Não det. & - \\
\hline & & $\begin{array}{l}\text { Redonda } \\
\text { (Figura 33) }\end{array}$ & Verde & Ausente & 1 & Cecidomyiidae & $\begin{array}{l}\text { Formicidae sp. } 2 \\
\text { Eulophidae }\end{array}$ \\
\hline
\end{tabular}


Esses inquilinos pertencem a duas espécies de Coleoptera, uma de Lepidoptera e uma de Thysanoptera. (Tabela 2).

Foram encontradas formigas predadoras (Hymenoptera: Formicidae) em um único morfotipo de galhas, e ácaros e Psocoptera sucessores em três morfotipos (Tabela 2).

\section{Discussão}

O PEPCV mostrou menor riqueza de galhas de insetos que as demais restingas investigadas no estado do Rio de Janeiro e São Paulo (RJ: 43 morfotipos em Grumari e 108 em Maricá e Carapebus; SP: 233 em Bertioga) (Oliveira \& Maia 2005; Maia 2001, Maia et al. 2008). No entanto, a média de 1,8 morfotipos de galhas por espécie de planta hospedeira foi similar aos valores foram encontrados para Grumari $(1,7)$ (Oliveira \& Maia 2005), Maricá (1, 9) (Maia 2001) e Bertioga $(1,9)$ (Maia et al. 2008). Para o Parque Nacional de Jurubatiba (RJ), a média foi um pouco mais alta (2,5) (Monteiro et al. 2004).

Houve diferenças na riqueza dos morfotipos de galhas em diversas espécies de plantas hospedeiras que também ocorrem em outras restingas (Figura 4), sendo menor em Cordia verbenacea DC (Boraginaceae); Guapira opposita (Vell.) Reitz (Nycyaginaceae); Neomitranthes obscura Sobral \& Zambom (Myrtaceae) e Protium icicariba (DC.) Marchand (Burseraceae) e maior em Clusia hilariana Schltdl (Clusiaceae), Guapira pernambuscensis (Casar.) Lundell (Nyctaginaceae) e Ouratea cuspidata (A. St.-Hil.) Engl. (Ochnaceae).

Para Byrsonima sericea DC. (Malpighiaceae); Manilkara subsericea (Mart.) Dubard (Sapotaceae); Myrciaria floribunda (H. West ex Willd.) Legrand (Myrtaceae); Ocotea notata (Nees) Mez (Lauraceae); Smilax rufescens Griseb. (Smilacaceae) e Couepia ovalifolia (Schott) Benth (Chrysobalanaceae), o número de morfotipos de galhas registrados em PEPCV encontra-se dentro dos valores assinalados para outras restingas (de 2 a 4 na primeira espécie, de 1 a 4 nas três seguintes, de 2 a 3 em
S. rufescens e 2 na última) (Oliveira \& Maia 2005; Maia 2001, Maia et al. 2008; Monteiro et al. 1994 e Monteiro et al. 2004).

Não foi observada a ocorrência de galhas de insetos em Cactaceae e Araceae, embora tais famílias de plantas apresentem esses tumores em outras áreas de restinga (Cactaceae em Maricá, RJ e Araceae em Bertioga, SP). Tampouco foram encontradas galhas em Bromeliaceae e Arecaceae, famílias até o momento sem registro de galhas em restingas.

As diferenças na composição das plantas hospedeiras e riqueza de galhas no PEPCV podem resultar de diferenças tanto na composição florística dessa restinga, como no método de amostragem. Deve-se ressaltar que, no PEPCV, embora a área investigada tenha sido menor, três espécies de plantas (Clusia hilariana, Guapira pernambuscensis e Ouratea cuspidata) apresentaram um número maior de morfotipos de galhas que em outras restingas (Figura 4).

Nenhum morfotipo foi observado em flores, raízes aéreas e gavinhas, diferindo das restingas do estado do Rio e de São Paulo para as quais há registros de galhas nessas partes da planta. E cada morfotipo de galha ocorreu em um único órgão da planta hospedeira, diferindo mais uma vez das restingas do estado do Rio de Janeiro e de São Paulo, onde foi verificada a ocorrência de alguns morfotipos em mais de um órgão da planta.

A maioria das galhas ocorreu em folhas e o segundo órgão mais atacado foi o caule/ gema (Figura 1). Em todos os estudos anteriores desenvolvidos em áreas de restinga, foi assinalada a predominância de galhas em folhas, seguidas por galhas caulinares ou das gemas. Maia (2001) sugeriu que essa alta incidência em folhas possa ocorrer em função das mesmas representarem um recurso abundante e constante em restingas. Esse resultado corrobora o padrão apontado por Mani (1964) para a América do Sul, onde aproximadamente 70\% dos registros são de galhas foliares.

Com relação à morfologia externa e interna das galhas, verificamos o predomínio de galhas verdes, glabras e uniloculares. Essa mesma predominância ocorre nas outras restingas. Quanto à forma, algumas diferenças

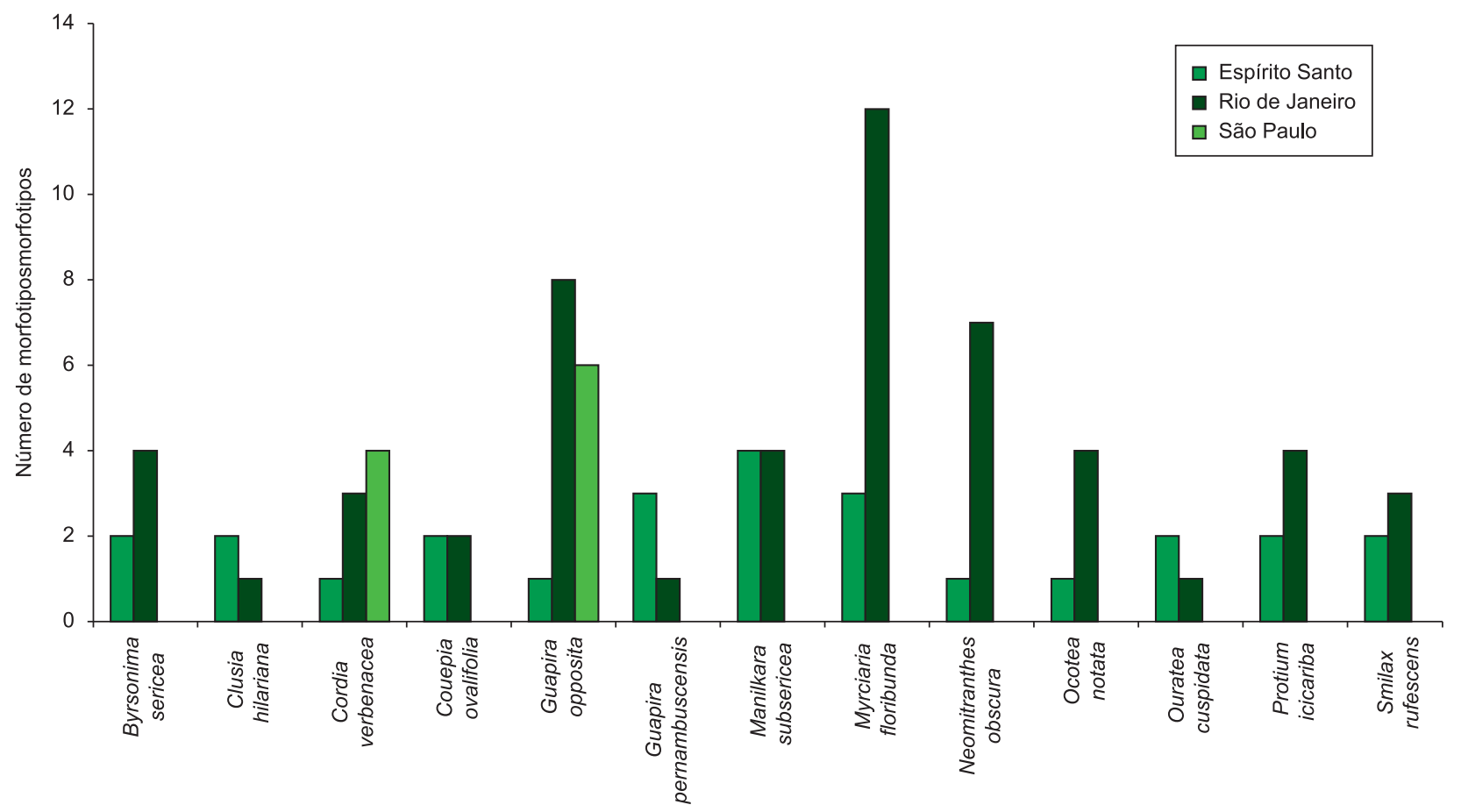

Figura 4. Número de morfotipos de galhas de insetos encontradas em 13 espécies de plantas hospedeiras nas restingas do Rio de Janeiro, Espírito Santo e São Paulo.

Figure 4. Number of morphotypes of insect galls found on 13 species of host plant in restingas of Rio de Janeiro, Espírito Santo and São Paulo. 

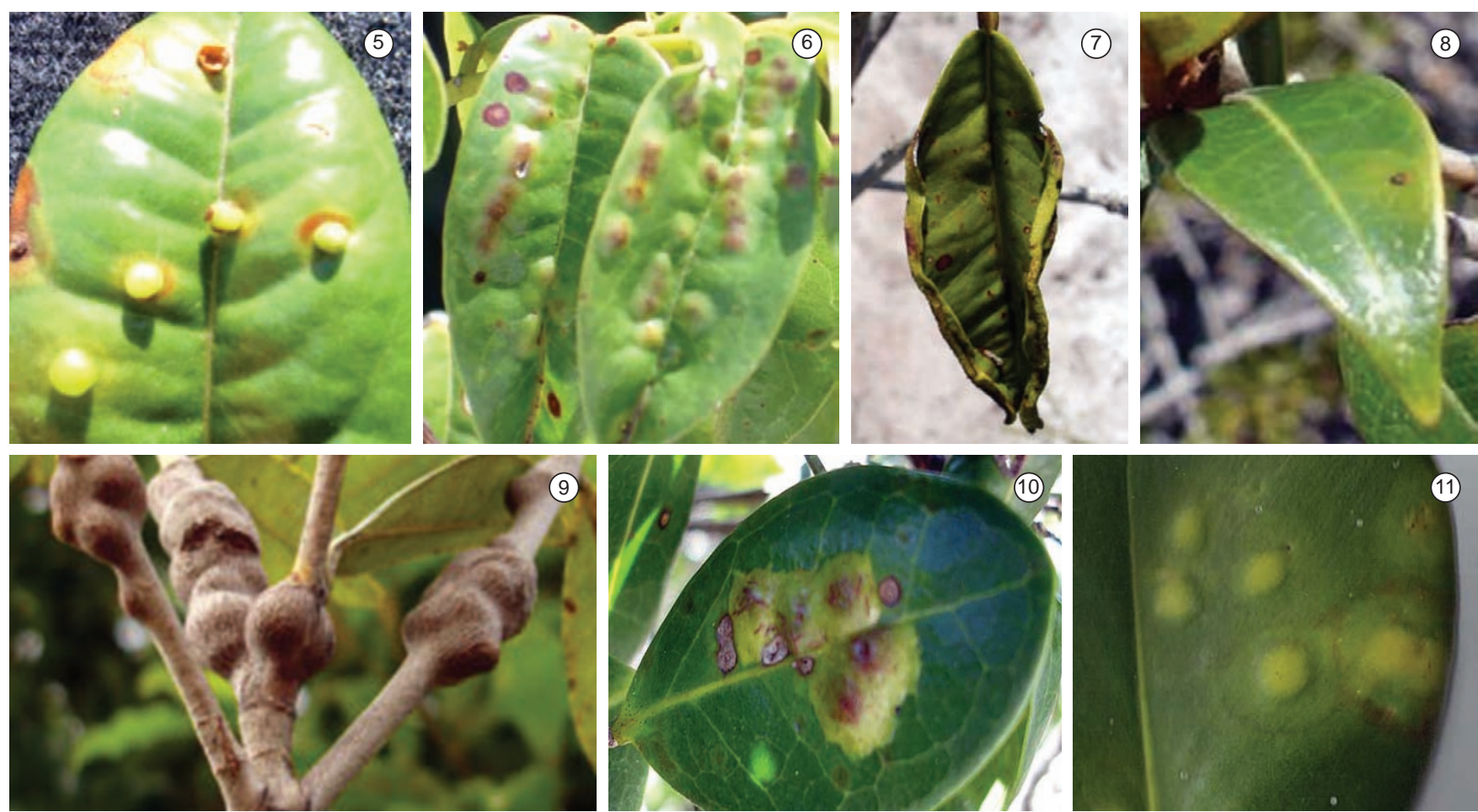

(11)
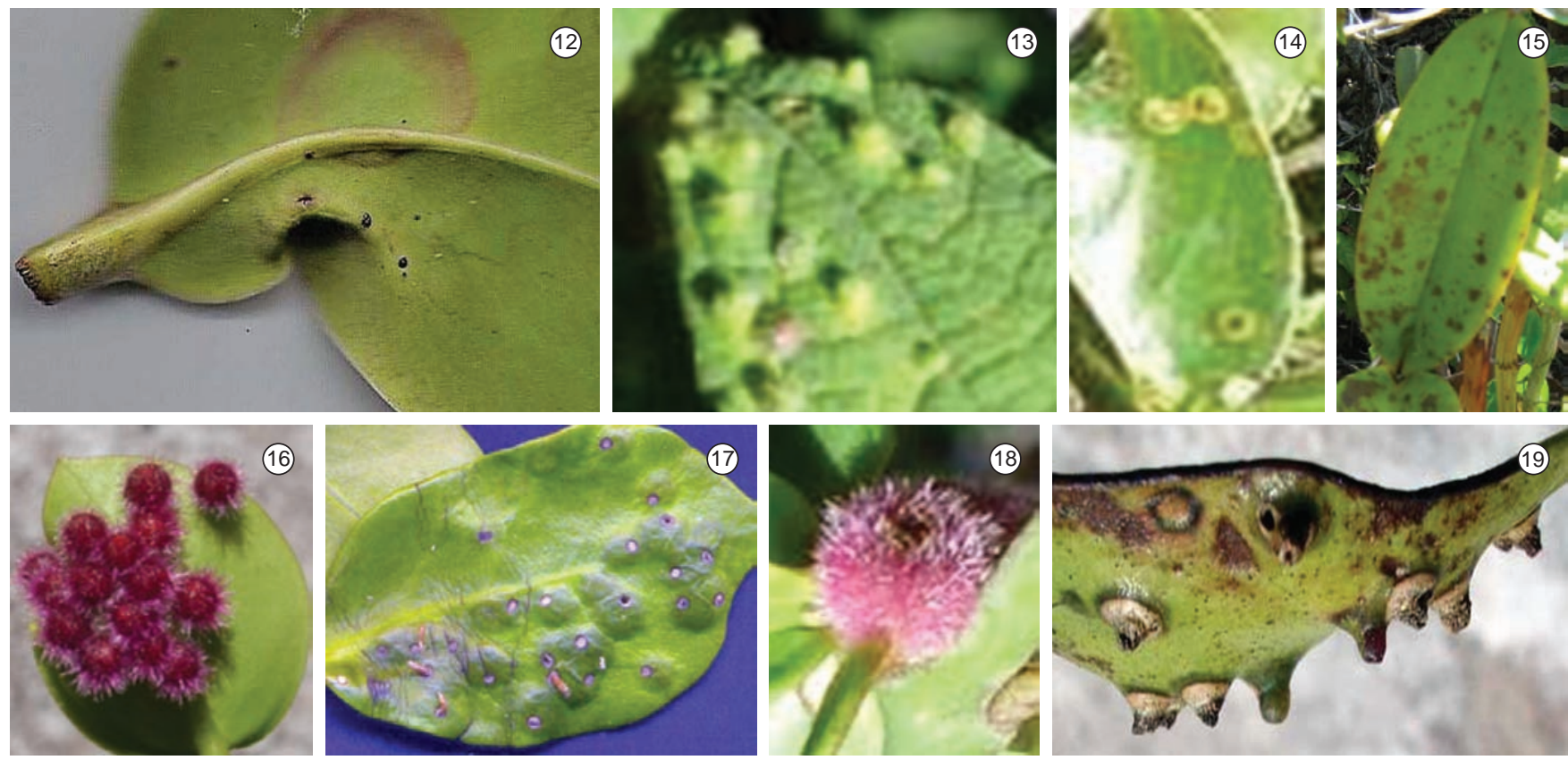

Figuras 5-19. Galhas de insetos do Parque Estadual Paulo César Vinha (E.S.). 5-7) em Andira nitida: 5) globosa foliar; 6) circular foliar; 7) enrolamento marginal da folha; 8-9) em Byrsonima sericea: 8) circular foliar; 9) globosa caulinar; 10) em Chaetocarpus myrsinites: esférica foliar; 11-12) em Clusia hilariana: 11) circular foliar; 12) enrolamento marginal da folha; 13) em Cordia verbenacea: globosa foliar; 14) em Couepia ovalifolia: esférica foliar; 15) em Epidendrum denticulatum: circular foliar; 16-18) em Guapira pernambuscensis: 16) globosa foliar; 17) esférica foliar; 18) globosa caulinar; 19) em Guapira sp., cônica foliar.

Figures 5-19. Insect galls in the Parque Estadual Paulo César Vinha (E.S.). 5-7) on Andira nitida: 5) globose leaf gall; 6) circular leaf gall; 7) marginal leaf roll; 8-9) on Byrsonima sericea: 8) circular leaf gall; 9) globose stem gall; 10) on Chaetocarpus myrsinites: spherical leaf gall; 11-12) on Clusia hilariana: 11) circular leaf gall; 12) marginal leaf roll; 13) Cordia verbenacea: globose leaf gall; 14) on Couepia ovalifolia: spherical leaf gall; 15) on Epidendrum denticulatum: circular leaf gall; 16-18) on Guapira pernambuscensis: 16) globose leaf gall; 17) spherical leaf gall; 18) globose stem gall; 19) on Guapira sp., conical leaf gall.

podem ser assinaladas. No PEPCV, as galhas globosas, enrolamentos da borda foliar e galhas redondas foram mais comuns; em Grumari, as fusiformes e circulares; em Maricá, as ovóides, os enrolamentos marginais e as circulares e em Bertioga, as fusiformes, globosas, circulares e ovóides predominaram. Devemos ressaltar, no entanto, que não há uma padronização na terminologia das formas, e os termos usados nem sempre são precisos. Galhas globosas, redondas e esféricas podem ser empregadas por diferentes autores para designar a mesma forma. 

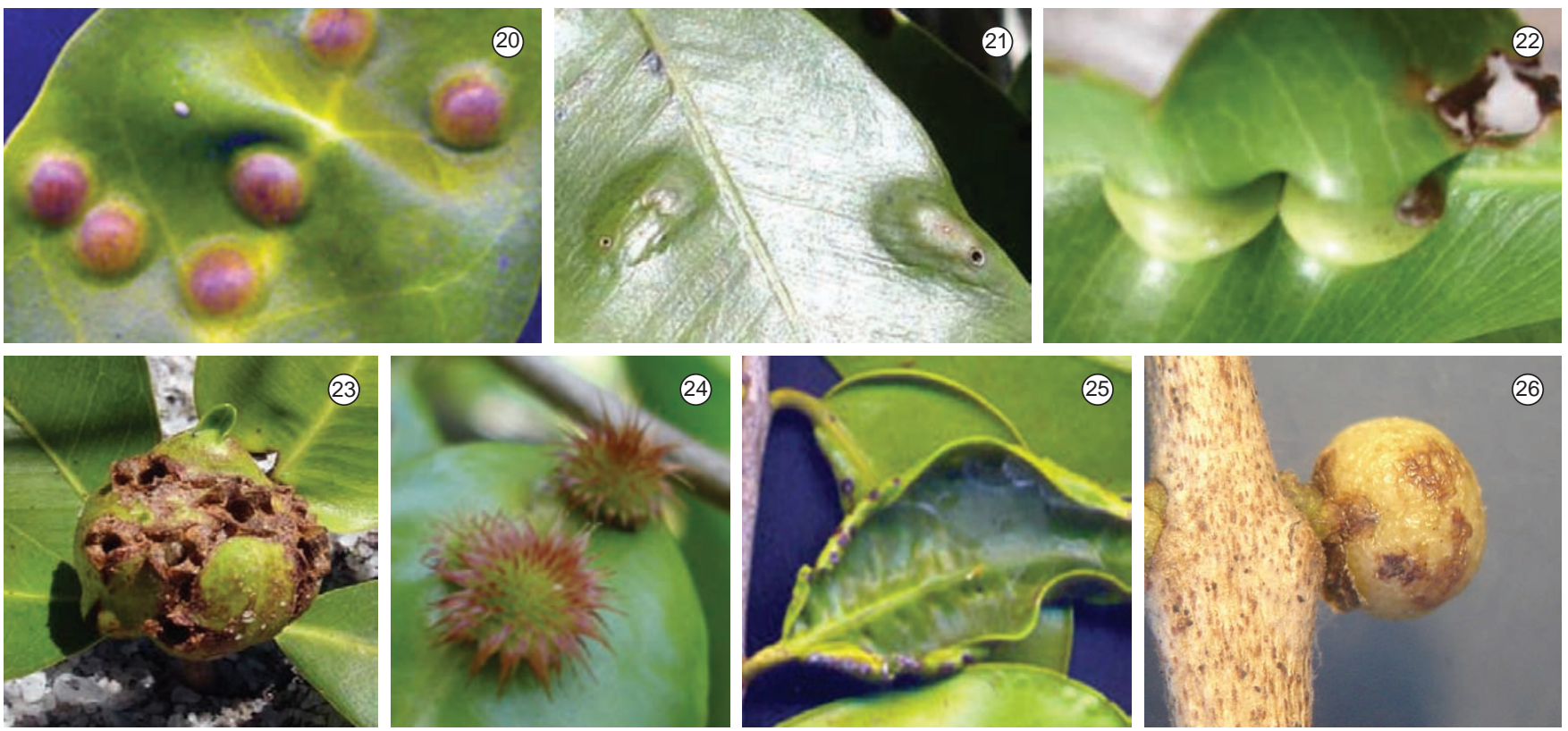

(26)
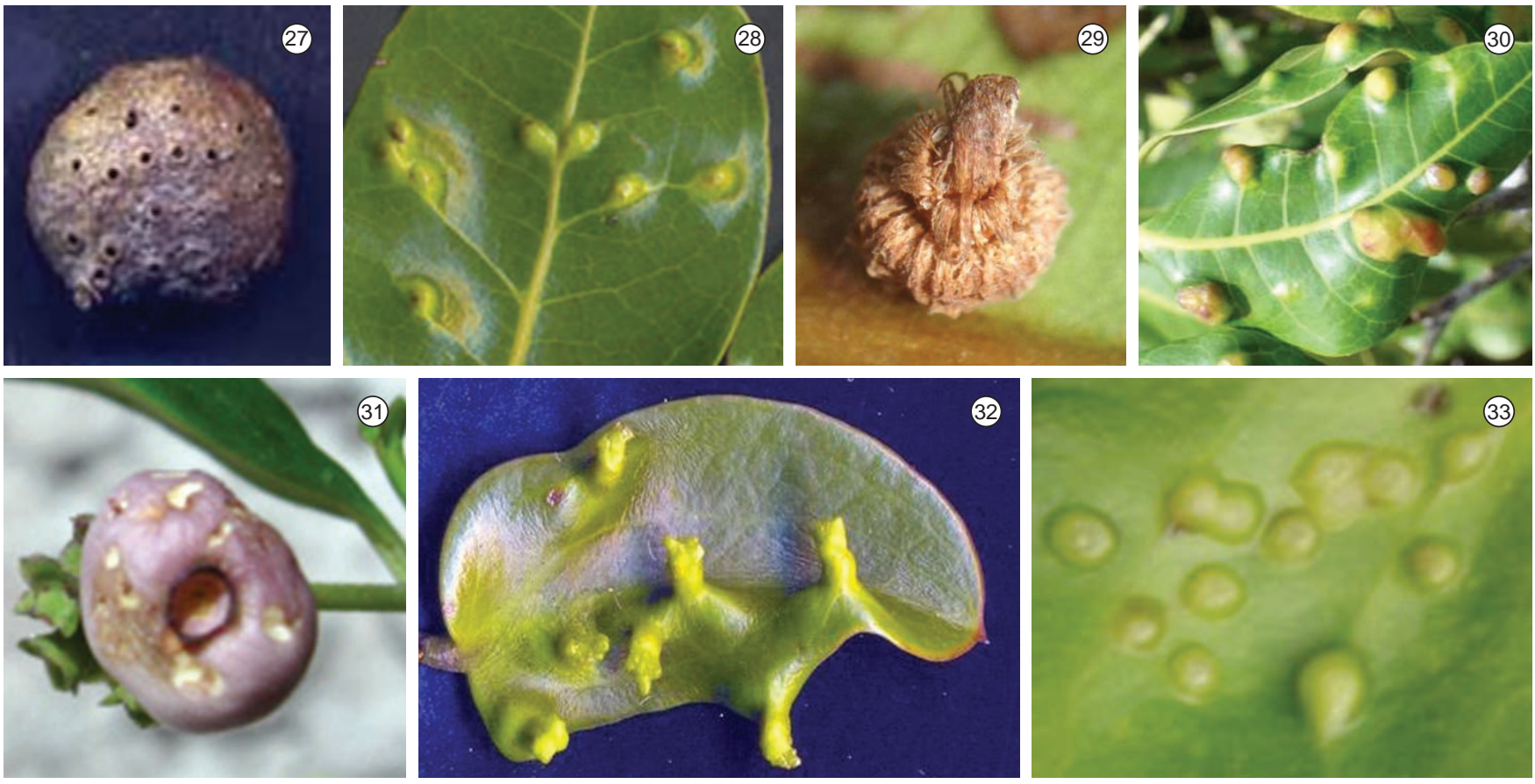

Figuras 20-33. Galhas de insetos do Parque Estadual Paulo César Vinha (E.S.). 20) em Ilex sp.: esférica foliar; 21-23) em Manilkara subsericea: 21) elíptica foliar; 22) esférica foliar; 23) globosa (gema); 24-26) em Myrciaria floribunda: 24) globosa foliar; 25) enrolamento marginal da folha; 26) globosa (gema); 27) em Myrtaceae: globosa (gema); 28) em Ocotea notata: esférica foliar; 29) em Ouratea cuspidata: 29) triangular foliar; 30) em Protium icicariba: esférica foliar; 31) em Salzmania nitida: globosa (fruto); 32-33) em Smilax rufescens: 32) cônical foliar; 33) esférica foliar.

Figures 20-33. Insect galls in the Parque Estadual Paulo César Vinha (E.S.). 20) on Ilex sp.: spherical leaf gall; 21-23) on Manilkara subsericea: 21) elliptical leaf gall; 22) spherical leaf gall; 23) globose bud gall 24-26) on Myrciaria floribunda: 24) globose leaf gall; 25) marginal leaf roll; 26) globose bud gall; 27) on Myrtaceae: globose bud gall; 28) on Ocotea notata: spherical leaf gall; 29) on Ouratea cuspidata: 29) triangular leaf gall; 30) on Protium icicariba: spherical leaf gall; 31) on Salzmania nitida: globose fruit gall; 32-33) on Smilax rufescens: 32) conical leaf gall; 33) spherical leaf gall.

As galhas foram induzidas por Diptera (Cecidomyiidae) e Hemiptera. Não foram encontradas galhas induzidas por Thysanoptera, Lepidoptera, Coleoptera e Hymenoptera. Comparando-se a guilda de galhadores do PEPCV com a de outras restingas, verificamos que, no referido Parque, ela é menos diversificada. Nas restingas do estado do Rio de Janeiro, essa guilda é composta por representantes de seis ordens de insetos e em São Paulo por cinco. Todos os Diptera galhadores pertencem à família Cecidomyiidae. Nas restingas do estado do Rio, o mesmo resultado foi encontrado. Diferentemente, em São Paulo, foram encontrados representantes de mais uma família: Tephritidae (Maia et al. 2008).

Seis cecidomiídeos galhadores foram identificados em nível de espécie, dois em nível de gênero e os demais em família. Para as restingas do estado do Rio de Janeiro, o conhecimento taxonômico desses galhadores é muito maior, em função do maior tempo de estudo da fauna local: 66 estão identificados em nível de espécies, 20 em gênero e o restante em categorias supra genéricas. Já para São Paulo, o conhecimento ainda é incipiente: 29 estão identificados em 
nível de espécie, 41 em nível genérico e os demais em categorias supra genéricas. As seis espécies de Cecidomyiidae encontradas no PEPCV ocorrem também nas restingas do estado do Rio de Janeiro, e apenas uma delas em São Paulo.

Dentre os hemípteros galhadores, apenas um foi identificado em nível de família, sendo os demais identificados em ordem. Para as restingas do estado do Rio de Janeiro, há três registros em família e o restante em ordem.

Conforme indicado nos estudos de Maia (2001), outros organismos além dos indutores podem ser encontrados no interior das galhas, atuando como inquilinos, predadores, sucessores e parasitóides. Maia (2001a) assinala como parte dessa fauna associada Pseudoscorpiones, Hymenoptera, Coleoptera, Lepidoptera e Diptera, e aponta os Hymenoptera como o grupo mais freqüente, sendo a maioria parasitóide, e alguns fitófagos.

A fauna de artrópodes associada às galhas no PEPCV incluiu colêmbolos, ácaros, Hymenoptera, Coleoptera, Lepidoptera, Thysanoptera, Diptera, Hemiptera e Psocoptera.

Com relação aos microhimenópteros parasitóides, PEPCV mostrou menor freqüência e menor riqueza do que as demais restingas estudadas (RJ: frequência de 68\% e 14 famílias representadas, Maia \& Azevedo 2009; SP: 48\% e oito famílias, Maia et al.2008; PEPCV: $31 \%$ e cinco famílias).

Os inquilinos também se mostraram menos freqüentes e menos diversificados no PEPCV (frequiência de $10 \%$ e representantes de três ordens: Coleoptera, Lepidoptera e Thysanoptera). Nas restingas do estado do Rio de Janeiro, os inquilinos ocorreram em 18 morfotipos (17,6\% do total), com representantes de Diptera (Cecidomyiidae e Sciaridae), Hymenoptera (Torymidae e Eulophidae), Lepidoptera (Oecophoridae) e Coleoptera (Curculionidae) (Maia 2001a). Em Bertioga, a freqüência foi maior (57 morfotipos, 24,4\%), e a guilda mais diversificada, com representantes de Diptera (Cecidomyiidae e Sciaridae), Lepidoptera, Coleoptera (Curculionidae), Hemiptera (Tingidae, Membracidae e Coccidae) e Thysanoptera (Maia et al. 2008).

Foram encontradas formigas predadoras (Hymenoptera: Formicidae) associadas a um único morfotipo de galhas. Para as restingas fluminenses, há registros de Lestodiplosis spp. (Diptera, Cecidomyiidae) e pseudoscorpiões como predadores em um e três morfotipos, respectivamente (Maia 2001a). Para São Paulo, formigas predadoras foram obtidas de dois morfotipos de galhas e Lestodiplosis spp. (Diptera: Cecidomyiidae) de três morfotipos (Maia et al. 2008). Portanto, a guilda dos predadores no PEPCV mostrou-se mais uma vez menos freqüente e diversificada.

Quanto aos sucessores, ácaros e Psocoptera foram encontrados em três morfotipos de galhas. Nas restingas do estado do Rio de Janeiro, tem-se registro apenas de formigas (Leptothorax sp.) colonizando galhas cilíndricas já abandonadas pelo indutor em Eugenia rotundifolia Casar (Myrtaceae). Já em São Paulo, foram encontrados Collembola,, Psocoptera e Thysanoptera como sucessores em sete, três e um morfotipo de galha, respectivamente (Maia et al. 2008).

Portanto, o PEPCV difere das restingas fluminenses e paulistas com relação à composição da fauna de invertebrados associada às galhas de insetos. Contudo, é preciso ressaltar que, na restinga capixaba, o esforço amostral foi menor em relação ao período de coleta e ao tamanho das áreas amostradas e que a metodologia investigativa utilizada não foi a mesma.

\section{Conclusões}

O estudo corrobora as Nyctaginaceae e Myrtaceae e aponta as Sapotaceae como as famílias de planta com maior riqueza de morfotipos de galhas de insetos em restingas.

Foi assinalada, pela primeira vez, a ocorrência de galhas em Ilex sp., Andira nitida, Chaetocarpus myrsinites, Salzmania nitida e Epidendrum denticulatum.
Lopesia simplex, Dasineura couepiae, Clusiamyia granulosa, Dasineura myrciariae, Manilkaramyia notabilis e Cordiamyia globosa registradas pela primeira vez para o estado do Espírito Santo, tiveram sua área de distribuição geográfica aumentada. A distribuição previamente conhecida incluía apenas o estado do Rio de Janeiro.

A distribuição das galhas nos órgãos vegetais seguiu o padrão já conhecido para restingas, com predomínio de galhas foliares, seguidas das caulinares e da gema.

Com relação à morfologia, galhas redondas, globosas e enrolamentos da borda foliar foram mais comuns. A maioria das galhas apresentou coloração igual a do órgão de ocorrência, com predomínio da cor verde; uma única câmara interna e ausência de pêlos, características morfológicas também predominantes em outras restingas.

Apenas duas ordens de insetos galhadores foram encontradas: Diptera (Cecidomyiidae) e Hemiptera, com claro predomínio dos Cecidomyiidae. Microhimenópteros parasitóides ocorreram em doze morfotipos de galhas, estando representados por cinco famílias; inquilinos em três morfotipos, estando representados por três ordens. E em um único morfotipo foram encontradas formigas predadoras (Hymenoptera). Ácaros e Psocoptera sucessores ocorreram, respectivamente, em um e dois morfotipos de galhas. A guilda de galhadores e a fauna de artrópodes associada às galhas mostraramse menos diversificadas e freqüentes no PEPCV que nas demais restingas investigadas, no entanto devem-se considerar as diferenças na metodologia amostral.

\section{Agradecimentos}

Ao CNPQ (Proc. 472084/2007-0 e 301197/2007-5) pelo apoio financeiro e ao Dr. Marcelo Tavares (Universidade Federal do Espírito Santo) pela identificação dos Hymenoptera.

\section{Referências}

ALMEIDA, L.M., RIBEIRO-COSTA, C.S. \& MARINONI, L. 2003. Manual de coleta, conservação, montagem e identificação de insetos. Holos, Ribeirão Preto, 78 p.

ARAÚJO, D.S.D. \& HENRIQUES, R.P.B. 1984. Análise florística das restingas do estado do Rio de Janeiro. In Restingas: origem, estrutura, processos (L.D. Lacerda, D.S.D. Araújo, R. Cerqueira \& B. Turcq, eds.). CEUFF, Niterói, p. 159-193.

BROWER, J.E. \& ZAR, J.H. 1984. Field and laboratory methods for general ecology. Wm.C. Brown Publishers, Boston, 226 p.

FABRIS, L.C. 1995. Composição florística e fitossociologia de uma faixa de floresta arenosa litorânea do Parque Estadual de Setiba, Município de Guarapari, ES. Dissertação de Mestrado, Universidade Estadual Paulista, Rio Claro.

FIDALGO, O. \& BONONI, V.L.R. 1989. Técnicas de coleta, preservação e herborização de material botânico. Instituto de Botânica, São Paulo, p. 34-38.

GAGNÉ, R.J. 1994. The gall midges of the Neotropical Region. Cornell University Press, Ithaca, 364 p.

MAIA, V.C. 2001. The gall midges (Diptera, Cecidomyiidae) from three restingas of Rio de Janeiro State, Brazil. Rev. Bras. Zool. 18(2):583-629.

MAIA, V.C., MAGENTA, M.A.G. \& MARTINS, S.E. 2008. Ocorrência e caracterização de galhas de insetos em áreas de restinga de Bertioga (São Paulo, Brasil). Biota Neotrop. 8(1):http:/www.biotaneotropica. org.br/v8n $1 / \mathrm{pt}$

MAIA, V.C. \& AZEVEDO, M.A.P. 2009. Diversidade de micro-himenópteros associados com galhas de Cecidomyiidae (Diptera) em restingas do estado do Rio de Janeiro (Brasil). Biota Neotrop. 9(2):151-164.

MANI, M.S. 1964. Ecology of plant galls. Dr. W. Junk Publisher, Holanda, $434 \mathrm{p}$. 
MONTEIRO, R.F., FERRAZ, F.F.F., MAIA, V.C. \& AZEVEDO, M.A.P. 1994. Galhas entomógenas em restingas: uma abordagem preliminar. In Anais do III Simpósio de Ecossistemas da Costa Brasileira: subsídios a um gerenciamento ambiental (S. Watanabe, coord.). ACIESP, São Paulo, p. 210-220. (v. 3)

MONTEIRO, R.F., ODA, R.A.M., NARAHARA, K.L. \& CONSTANTINO, P.A.L. 2004. Galhas: diversidade, especificidade e distribuição. In Pesquisas de longa duração na Restinga de Jurubatiba: ecologia, história natural e conservação (C.F.D. Rocha, F.A. Esteves \& F.R. Scarano, orgs.). Rima, São Carlos, 376 p.

NEIMAN, Z. 1989. Era verde? Ecossistemas brasileiros ameaçados. Atual, São Paulo, p. 80-81.
OLIVEIRA, J.C. \& MAIA, V.C. 2005. Ocorrência e caracterização de galhas de insetos na restinga de Grumari (Rio de Janeiro, RJ, Brasil). Arq. Mus. Nac. 63(4):669-675.

OLIVEIRA, J.C. 2009. Viabilidade de espécies galhadoras (Diptera, Cecidomyiidae) e parasitóides (Hymenoptera) associadas à Guapira opposita (Vell.) (Nyctaginaceae) como bioindicadores da qualidade ambiental. Tese de Doutorado, Universidade Federal do Rio de Janeiro, Rio de Janeiro.

Recebido em 07/10/09

Versão reformulada recebida em 11/12/10

Publicado em 08/02/10 